\title{
Optical spectroscopy on Monomeric and Polymeric 1:1 Fulleride Salts
}

\author{
K. Kamarás, ${ }^{1}$ D.B. Tanner, ${ }^{2}$ L. Forró,${ }^{3}$ M.C. Martin,${ }^{4}$ L. Mihaly, ${ }^{4}$ H. Klos,${ }^{5}$ and \\ B. Gotschy
}

Received 5 January 1995

We compare infrared spectra of the $\mathrm{C}_{60}^{-}$monoanion in different solid-state structures with each other as well with that of the neutral molecule. We relate the shift and splitting of the $T_{u}$ infrared modes to the strength and anisotropy of electron-phonon coupling in those environments.

KEY WORDS: Fullerenes; optical properties; polymers

\section{INTRODUCTION}

$\mathrm{C}_{60}$, the prime example of the recently discovered fullerenes, tends to form ionic salts by attracting up to 6 electrons. Of these, the trianions are the most extensively studied due to the $\mathrm{A}_{3} \mathrm{C}_{60}$ superconducting compounds, where $\mathrm{A}$ is an alkali atom. However, intriguing solid-state effects can be found in other phases as well. The most recent examples are the alkali salts with 1:1 composition. These materials undergo several phase transitions depending on thermal history. There is growing evidence for an orthogonal phase (ortho-I) consisting of covalently bound polymers [1,2] and another one (ortho-II) of dimers [3]. Vibrational spectroscopy has been used to identify the valence state of anions in $\mathrm{C}_{60}$ salts $[4,5]$ and it was shown early on that electron-phonon interactions play a significant role in the infrared properties of the salts [6]. In this study, we compare the infrared spectra of different phases of $\mathrm{RbC}_{60}$ to $\left(\mathrm{Ph}_{4}\right)_{2} \mathrm{C}_{60} \mathrm{I}$, a charge transfer salt containing $\mathrm{C}_{60}^{-}$monoanions.

\footnotetext{
${ }^{1}$ Research Institute for Solid State Physics, Budapest, Hungary H 1525

${ }^{2}$ Dept. Physics, University of Florida, Gainesville, FL 32611

${ }^{3}$ Departement de Physique, École Polytechnique Féderale de Lausanne, Lausanne, Switzerland CH-1015

${ }^{4}$ Department of Physics, SUNY at Stony Brook, Stony Brook, NY 11794

${ }^{5}$ University of Bayreuth, Germany D 95440
}

\section{EXPERIMENTAL}

$\left(\mathrm{Ph}_{4}\right)_{2} \mathrm{C}_{60} \mathrm{I}$ crystals were grown electrochemically [7]. The process results in a composition which ensures -1 charge on the fullerene ball. $\mathrm{RbC}_{60}$ powder was prepared by reacting $\mathrm{C}_{60}$ and $\mathrm{Rb}$ at high temperature. Infrared spectra of the tetraphenylphosphonium salt crystals were taken under an infrared microscope in reflectance mode at room temperature; the rubidium compound was ground into $\mathrm{KCl}$ pellets and the transmission measured against a pure $\mathrm{KCl}$ reference at each temperature. The room-temperature stable phase of $\mathrm{RbC}_{60}$ is the ortho-I structure; it transforms to fcc above $400 \mathrm{~K}$ and from there can be quenched into the ortho-II form which is metastable but has a long relaxation time when kept under $250 \mathrm{~K}$.

Unlike the superconducting salts or the ferromagnetic $\mathrm{TDAE}_{-} \mathrm{C}_{60}$, both our materials are stable in air thus requiring no special precautions during measurements; a slight decomposition into $\mathrm{C}_{60}$ is observed when heating $\mathrm{RbC}_{60}$ but this does not affect our main conclusions.

\section{RESULTS AND DISCUSSION}

Fig. 1. shows the section of the infrared spectrum containing the two high-frequency infraredactive vibrations of $\mathrm{C}_{60}$. (To facilitate the comparison, we present the data as absorption, calculated by Kramers-Kronig analysis of the reflectance and as 
$A=-\log T$ from transmission.) The two modes in question are found at 1183 and $1429 \mathrm{~cm}^{-1}$, respectively, in pure $\mathrm{C}_{60}$.

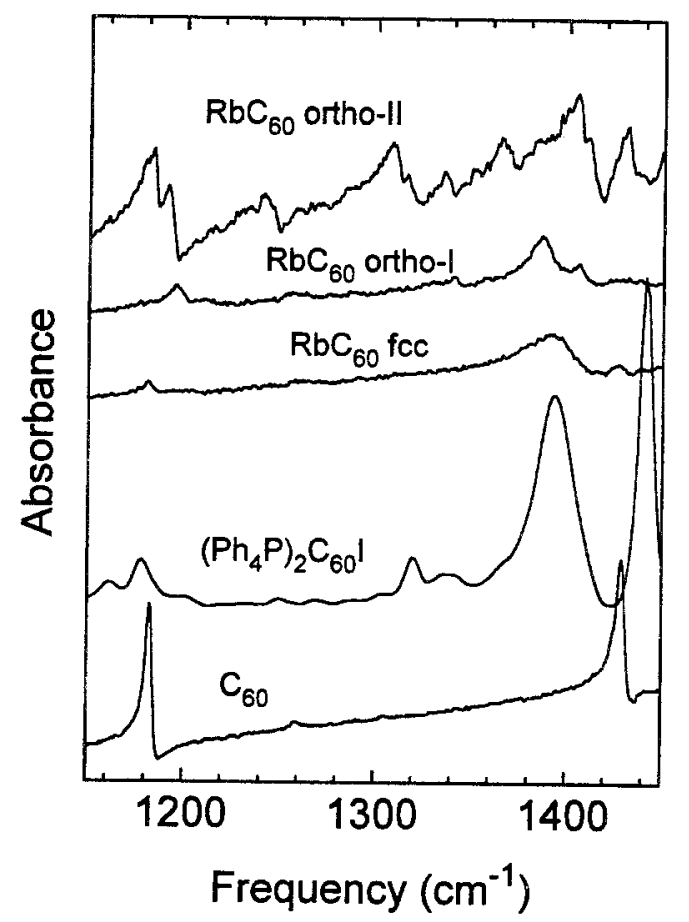

Fig. 1. Infrared spectra of $\mathrm{C}_{60},\left(\mathrm{Ph}_{4}\right)_{2} \mathrm{C}_{60} \mathrm{I}$ and the fcc, ortho-I and ortho-II phases of $\mathrm{RbC}_{60}$.

The somewhat complicated frequency shifts of the two $\mathrm{T}_{u}$ modes can be understood taking into account two solid-state effects: the change in lattice constant and the electron-phonon interaction. If the distance between fulleride ions gets shorter, the modes should harden in absence of other perturbations; electron-phonon interaction, on the other hand, causes softening whose magnitude depends on the strength of the coupling. As shown both experimentally $[4,5]$ and theoretically $[6]$, the highestfrequency mode has a much larger electron-phonon coupling constant than the $1183 \mathrm{~cm}^{-1}$ mode. Therefore, the former is indicative of the coupling, while the latter reflects the changes in lattice constant.

In $\left(\mathrm{Ph}_{4}\right)_{2} \mathrm{C}_{60} \mathrm{I}$ the $\mathrm{C}_{60}$ ions are separated from each other by the bulky organic cations. Thus this compound can be regarded as a prototype of an isolated monoanion in a crystalline environment. The shift in the $\mathrm{T}_{u}(4)$ mode from 1429 to $1394 \mathrm{~cm}^{-1}$ results from the on-ball electron-vibrational interaction. (Note that there are several modes of the counterion present, however, the fullerene modes can be clearly identified.) This mode does not shift appre- ciably on going to the fcc phase, indicating that the fcc phase can also be regarded as an ensemble of $\mathrm{C}_{60}^{-}$ ions, interacting with the electron on the ball but not with each other. The ortho-I phase shows increased softening to $1385 \mathrm{~cm}^{-1}$, along with a splitting into three components, at 1340,1385 and $1406 \mathrm{~cm}^{-1}$ [5]. The softening can be related to an increased interaction with the collective electronic system and the splitting to the one-dimensional character of the polymeric chains, leading to the lifting of the threefold degeneracy of the $T_{u}$ modes. Finally, the orthoII phase has a definite reduction in symmetry, with many more modes showing, but the vibration in question shifting up in frequency, indicative of the lattice contraction being more important than the electronphonon coupling.

In contrast, the position of the $\mathrm{T}_{u}(3)$ mode at $1183 \mathrm{~cm}^{-1}$ is determined by structural effects. In the fcc phase this peak is at the same position as in neutral $\mathrm{C}_{60}$. The slight downshift in the phosphonium salt reflects the increased distance between fullerenes in that lattice. The significant contraction in the orthorombic phases leads to a hardening in both ortho-I and ortho-II $\mathrm{RbC}_{60}$. As expected, the hardening is more pronounced in the ortho-I phase, which shows a stronger compression along the [110] direction.

\section{ACKNOWLEDGEMENTS}

Work supported by the U.S.-Hungarian Joint Fund Grant No. 271, the Bundesminister für Forschung und Technologie (Berlin, Germany) and by the European Community Grant No. CIPA-CT93-0032.

\section{REFERENCES}

1. S. Pekker, A. Jánossy, L. Mihály, O. Chauvet, M. Carrard, and L. Forró, Science 265, 1077 (1994).

2. P. W. Stephens, G. Bortel, G. Faigel, M. Tegze, A. Jánossy, S. Pekker, G. Oszlányi, and L. Forró, $\mathrm{Na}$ ture 370, 636 (1994).

3. G. Oszlányi, G. Bortel, G. Faigel, M. Tegze, L. Gránásy, S. Pekker, P. W. Stephens, G. Bendele, R. Dinnebier, G. Mihály, A. Jánossy, O. Chauvet, and L. Forró, Phys. Rev. $B$, submitted.

4. M. C. Martin, D. Koller, X. Du, P. W. Stephens, and L. Mihaly, Phys. Rev. B 49, 10818 (1994).

5. T. Pichler, R. Winkler, and H. Kuzmany, Phys. Rev. B 49, 15879 (1994).

6. M. J. Rice and H.-Y. Choi, Phys. Rev. B 45, 10173 (1992).

7. M. Keil, H. Klos, A. Schilder, W. Schütz and B. Gotschy, Proceedings of IWEPNM' 94 , World Scientific, Singapore, to be published. 